\title{
Bioluminescence in a Complex Coastal Environment: 2. Prediction of Bioluminescent Source Depth From Spectral Water-leaving Radiance
}

\author{
Matthew J. Oliver \\ Coastal Ocean Observation Laboratory, Institute of Marine and Coastal Sciences, Rutgers \\ University, \\ New Brunswick, New Jersey, USA \\ Mark A. Moline \\ Biological Sciences Department, California Polytechnic State University, \\ San Luis Obispo, California, USA \\ Curtis D. Mobley and Lydia Sundman \\ Sequoia Scientific Inc., \\ Bellevue, Washington, USA \\ Oscar M. E. Schofield \\ Coastal Ocean Observation Laboratory, Institute of Marine and Coastal Sciences, Rutgers \\ University, \\ New Brunswick, New Jersey, USA
}

\section{Abstract}

Many bioluminescence observations are made from the ocean's surface. However, the depth of the bioluminescent source is difficult to estimate on the basis of surface observations alone, given the variable light attenuation of unknown concentrations of water column constituents such as phytoplankton, colored dissolved organic matter, and detritus. Part 1 of this paper showed that bioluminescent water-leaving radiance signals are detectable, even in extremely turbid and dynamic coastal waters. Here, in part 2 of this paper, we analyze the waterleaving radiance patterns of bioluminescence modeled by HydroLight 4.2 to determine if the depth of the bioluminescent source can be estimated from its spectral signature. We find that the depth of the bioluminescent source is contained within the spectral signal and can be elucidated by simple neural networks. These networks can predict the depth of a bioluminescent layer with great accuracy, solely on the basis of the spectral shape of bioluminescent water-leaving radiance in a variety of water column and bottom type conditions. In addition, we found that as little as three wavelengths from the spectrum of water-leaving radiance are sufficient for an accurate determination of the depth of the bioluminescent source.

Keywords: bioluminescence, radiative transfer, prediction.

\section{Introduction}


Bioluminescence in the marine environment is caused by a wide array of organisms, including dinoflagellates, ostracods, copepods, and coelenterates [Herring, 1987]. It is casually observed as a surface phenomenon after bioluminescent organisms have been mechanically disturbed by crashing waves, boat propellers or other swimming megafauna. The duration and pattern of the bioluminescent flash are often indicative of which bioluminescent taxa are present. For example, in the presence of mechanical disturbance, dinoflagellate bioluminescence often appears as a nearly constant low level glow, whereas a disturbed coelenterate exhibits relatively intense bursts of light.

Bioluminescence appears to serve a wide variety of ecological functions in the marine environment, from predator avoidance to mate attraction [Abrahams and Townsend, 1993; Burkenroad, 1943; Morin, 1983]. For example, it is thought that when bioluminescent dinoflagellates are attacked by a predator, their bioluminescent response serves to alarm secondary predators that prey upon the dinoflagellate attacker [Burkenroad, 1943]. In addition to ecological functions, light production as a function of mechanical stimulation provides a direct visual method of identifying surface and subsurface vessels and swimmer tracks. The development of a passive method (as opposed to active methods such as RADAR or LIDAR) to identify hostile ships, submarines, and swimmers, as well as the development of strategies that reduce the risk of detection are relevant [Lapota, 2003, 2005; Lynch, 1981]. Support for marine bioluminescence research in the last 40 years reflects this relative importance [Nealson, 1993]. Therefore the depth distribution of bioluminescent organisms is of ecological as well as strategic importance.

The intensity and spectral shape of an in-water light source observed at the surface is related to the total concentration of light attenuating constituents of the water column [Gordon, 1987; Leathers et al., 1999]. Phytoplankton, colored dissolved organic matter, and detritus all differentially absorb and scatter light; thus their distribution in relation to the light source is important in modulating the spectral shape of the bioluminescent water-leaving radiance $\left(\mathrm{BL}_{\mathrm{w}}\right)$. Theoretically, it is possible to use radiative transfer theory to derive the depth of the underwater light source, given sufficient information about the in situ attenuating constituents. However, this is a difficult analytical problem to solve without in-depth knowledge of the inherent optical properties (IOPs) of the water column [McCormick, 1992; Sundman et al., 1998].

Rather than solving for the depth of the internal light source analytically, we have taken a more pragmatic, statistical approach based on neural networks. Neural networks have been employed successfully in situations where the response of interest is a complex function of a variety of predictors. These models statistically relate the response to the predictors, and do not rely the on exact mechanistic understanding of the relationship between the response and the predictors. We develop and test a neural network model that predicts the depth of a bioluminescent light source on the basis of the spectral characteristics of $\mathrm{BL}_{\mathrm{w}}$. We do this using the natural distribution of bioluminescence measured in the New Jersey coastal environment described in part 1 of this paper [Moline et al., 2007]. Our primary approach is to use the measured bioluminescent distribution and IOPs to calculate $\mathrm{BL}_{\mathrm{w}}$ using a radiative transfer model. Then, we analyze $\mathrm{BL}_{\mathrm{w}}$ with three questions in mind: (1) Can the depth of bioluminescent layers be predicted on the basis of spectral $\mathrm{BL}_{\mathrm{w}}$ ? (2) What effect does changing the IOPs and bottom type of the water column have on $\mathrm{BL}_{\mathrm{w}}$ ? (3) How many wavelengths in the $\mathrm{BL}_{\mathrm{w}}$ spectrum are 
needed for an accurate prediction of the depth of the source? Our primary interest is using the spectrum as a proxy for the vertical position of the source, rather than understanding the mechanistic relationship between the internal light source and its spectrum. In short, we find that the depth of bioluminescent layers can be accurately predicted from the spectral qualities of $\mathrm{BL}_{\mathrm{w}}$, and that as little as three wavelengths in the spectrum are sufficient for a robust source depth prediction.

\section{Materials and Methods}

\subsection{Environmental Data}

Water column profiles of absorption, attenuation, backscatter and bioluminescent potential were collected at the Long-Term Ecosystem Observatory (LEO) in New Jersey during the summers of 2000 and 2001. Both data sets were collected using robotic vertical profilers. The profilers were deployed $10 \mathrm{~km}$ offshore of the New Jersey coastline in $14 \mathrm{~m}$ of water at $39^{\circ} 27.41$ $\mathrm{N}, 74^{\circ} 14.75 \mathrm{~W}$. The profiler frames were anchored to the seafloor with instrument packages attached to a floating drogue which was depth controlled by an attached winch. These profilers provided temperature and salinity using a Seabird $911 \mathrm{CTD}$, absorption and attenuation meter at nine wavelengths $(412,440,488,510,532,555,650,676$ and $715 \mathrm{~nm})$ using a Wetlabs ac-9, backscatter/fluorometer at two wavelengths $(470 \mathrm{~nm}$ and $676 \mathrm{~nm})$ using a HOBI Labs HydroScat, and an estimate of bioluminescent potential (bioluminescent light emitted by mixing the water) using a bioluminescence BP [Herren et al., 2005; Moline et al., 2005]. A centrifugaltype impeller pump drives the water into an enclosed $500 \mathrm{~mL}$ chamber in the BP section, where a light-baffled Hamatsu H5783 photomultiplier tube measures stimulated bioluminescence between 300 and $650 \mathrm{~nm}$. The inside of the chamber is coated with a $0.075 \mathrm{~mm}$ flat white coating to maximize the amount of stimulated light measured by a PMT. While IOP data were collected continually during the experiments, bioluminescence data were only collected during nighttime profiles (2100-0500) local time. In 2000, the profiler was deployed for $\sim 15$ days logging 339 nighttime profiles while in 2001, it was deployed for $\sim 8$ days logging 132 nighttime profiles. For a detailed description of the instruments profilers and the natural distributions of the data collected, see the methods section and Figures 4 and 5 in part 1 [Moline et al., 2007] of this paper.

\subsection{HydroLight Model Runs}

\section{--- INSERT FIGURE 1 ---}

A new bioluminescence module was integrated into the HydroLight v. 4.2 model [Mobley and Sundman, 2001a, 2001b] that measured profiles of bioluminescent potential to be used as internal light sources in the HydroLight model code. This allowed us to estimate $\mathrm{BL}_{\mathrm{w}}$ at our study site. The model settings used measured $0.25 \mathrm{~m}$ binned absorption, attenuation, and backscatter, as well as measured wind speeds to estimate sea surface roughness. Model runs used pure water absorption values by Pope and Fry [1997]. The volume scattering function was from the measured bb/b according to Petzold [1972]. The HydroLight model computed a new spectral scattering phase function when the backscatter to total scatter ratio changed by more than 0.005 . 
Default atmospheric parameters (except wind speed) were used for all runs, with the user defined irradiance field input set to zero to simulate nighttime conditions. The model was layered into 1 $\mathrm{m}$ sections to simulate stimulation of the bioluminescent organisms on a realistic scale. These specific locations in the water column ( $1 \mathrm{~m}$ thick in this case) were sequentially "stimulated" in each model run, rather than the entire water column at once. This layering approach decomposed each bioluminescence profile into thirteen, $1 \mathrm{~m}$ sections and allowed for the calculation of surface $\mathrm{BL}_{\mathrm{w}}$ of the entire water column if only discrete portions of the water column were emitting bioluminescent light. In part 1 of this paper [Moline et al., 2007], it was clear that the deeper the internal light source, the more red-shifted its spectra (Figure 1). Therefore, in this analysis, we focused on the spectral shape of $\mathrm{BL}_{\mathrm{w}}$ as a predictor of the depth of the bioluminescent source. $\mathrm{BL}_{\mathrm{w}}$ spectra were normalized to their respective maximum values and related to the depth of the bioluminescent layer.

\section{--- INSERT FIGURE 2 ---}

In part 1 of this paper, we estimated $\mathrm{BL}_{\mathrm{w}}$ from the naturally occurring IOPs and bioluminescent potential using HydroLight. Measured IOPs were highly variable and quite large in magnitude [Moline et al., 2007, Figures 4 and 5]. Here, in part 2, we examine how various attenuating conditions and bottom types affect the shape of $\mathrm{BL}_{\mathrm{w}}$. We proportionally reduced the values of the measured IOPs to $50 \%, 10 \%$, and $1 \%$ of the natural values of measured IOPs at LEO, but did not modify the bioluminescent potential values we measured at the study site. By doing this, we maintained the natural variance of the natural environment, but reduced the effect of the IOPs on $\mathrm{BL}_{\mathrm{w}}$. In addition, for each of these different IOP conditions, we added a sea grass or a coral sand bottom to determine what effect different bottom types may have on spectral $\mathrm{BL}_{\mathrm{w}}$ (an option in the HydroLight model) (Figure 2). We also normalized the spectra for each of these conditions to their respective maximum values to determine how the shape of $\mathrm{BL}_{\mathrm{w}}$ was modulated by the different IOP conditions and bottom types. This study represents 44,940 HydroLight model runs in addition to the 7490 runs already discussed in part 1 of this paper [Moline et al., 2007].

\subsection{Neural Net Model Construction}

We constructed our neural net using the nnet function [cf. Venables and Ripley, 2002] in the program R [R Development Core Team, 2006] to use the normalized spectral $\mathrm{BL}_{\mathrm{w}}$ to predict the vertical location of the bioluminescent layer. On the basis of the normalized spectral waterleaving radiance, we used the neural nets to predict both the geometric depth $(\mathrm{m})$ of the bioluminescent source and the dimensionless optical depth $\tau$, where

\section{$r(\lambda)=\int c(\lambda, x) d s$}

\section{(1)}

$\mathrm{c}$ is beam attenuation $\left(\mathrm{m}^{-1}\right)$ and $\mathrm{z}$ is depth $(\mathrm{m}) . \tau$ is also wavelength dependent, so for any given water-leaving spectrum, the wavelength we calculated $\tau$ at was the same as the wavelength of maximum $\mathrm{BL}_{\mathrm{w}}$. The form of the neural net model used was a basic single layer model with inputs (normalized spectral $\mathrm{BL}_{\mathrm{w}}$ ) going to a single layer of neurons then to the outputs (geometric depth or $\tau$ ). Fundamental to a neural network model design is a training set on which 
the neurons "learn" how the depth (meters or $\tau$ ) of the bioluminescent light source relates to the spectral shape of the water-leaving radiance. Unless otherwise specified, all networks were trained on a random $50 \%$ of the available data from both years. As with any non-linear regression, there is a danger of "over-fitting" the model to the data. If there are too few neurons, the patterns in the data will not be captured, if there are too many, the neurons will fit the noise in the training data and will not be a good predictor of future data. Therefore we tested networks with one to fifteen neurons to determine how many neurons were needed for the computer to sufficiently learn the patterns of the data without over fitting it by analyzing how well a neural net could predict data it was not trained on. We found that a single hidden layer of only five neurons in the neural net model was sufficient.

For each of the eight different conditions shown in Figure 2, we constructed two neural network models; one to predict the source depths in geometric meters and another for $\tau$. Then, using these networks, we predicted the remaining $50 \%$ of the associated conditions in the data set. In addition, we used each of these trained networks to predict the geometric depth or $\tau$ in conditions that the neural network was not trained on. For example, a neural net was trained on spectra from a water column with $50 \%$ of the IOPs with a sea grass bottom and then used to predict the geometric depth or $\tau$ of a bioluminescent source on the basis of $\mathrm{BL}_{\mathrm{w}}$ from a water column with $10 \%$ IOPs with a coral sand bottom. Root mean squared error (RMS) was used to evaluate each of the models (Tables 1a and 1b). In addition, we also parsed the data by year; training the neural nets on the year 2000 data and attempted to predict the year 2001 data.

\subsection{Wavelength Reduction}

To assess the number of wavelengths needed for robust predictions of the bioluminescent source depth based on the spectral $\mathrm{BL}_{\mathrm{w}}$, we systematically reduced the number of wavelengths analyzed by the neural net. While maintaining the wavelength distribution even across the spectrum, we reduced the original 37 wavelengths of $\mathrm{BL}_{\mathrm{w}}$ to $19,10,5,3$, and 2 wavelengths. For each wavelength reduction step, alternate wavelengths were chosen from the previous step. There was no intentional biological or physical basis for choosing the wavelengths at each reduction step. For each wavelength reduction, we again assessed how well the neural net trained on the year 2000 data was able to predict the depth or $\tau$ of the bioluminescent layer in the year 2001 by calculating an RMS error.

\section{Results}

\subsection{Analysis of the Bioluminescent Water-Leaving Radiance}

The $\mathrm{BL}_{\mathrm{w}}$ modeled by HydroLight under natural conditions indicated that, given the distribution of the bioluminescence and the IOPs, the spectral shape of $\mathrm{BL}_{\mathrm{w}}$ was highly variable. Under natural conditions, the lower the total $\mathrm{BL}_{\mathrm{w}}$, the more the spectrum shifts toward red, indicating that absorption in the blue wavelengths by phytoplankton, CDOM and detritus significantly modulate the bioluminescent signal [Moline et al., 2007, Figure 8]. In addition, the wavelength of maximum water-leaving radiance ranged nearly $100 \mathrm{~nm}$ and it was strongly correlated to the depth of the bioluminescent source indicating that depth information was 
present within the spectral shape $\left(\mathrm{R}^{2}=0.80\right.$, Figure 1$)$. However, the errors in estimating the depth of any particular bioluminescent layer based on the wavelength of maximum $\mathrm{BL}_{\mathrm{w}}$ alone were too large for a practical prediction of the source depth. For example, if the wavelength of maximum $\mathrm{BL}_{\mathrm{w}}$ was $520 \mathrm{~nm}$, Figure 1 shows that its source could be between 6 and 11 meters. Because the range and magnitude of attenuation of the water column were relatively high (for

example: $\mathrm{c} 488 \sim 1.1-9 \mathrm{~m}^{-1}$ ), a strong correlation between the wavelength of maximum $\mathrm{BL}_{\mathrm{w}}$ and the depth of the source would be expected [Kirk, 1994].

\section{--- INSERT FIGURE 3 ---}

The reduction of the total IOPs to $50 \%, 10 \%$, and $1 \%$ of the natural conditions showed that in general, the lower the magnitude of the total IOPs of the water column, the smaller the wavelength range of maximum $\mathrm{BL}_{\mathrm{w}}$. However, at the $1 \%$ IOP level, the range of maximum emission more than doubles, due to the interaction of the bioluminescent light with the bottom. With the reduction of the total IOPs, downward emission from the bioluminescent source reflects off the coral sand or sea grass bottom, then travels upward and out of the water column. This downward emitted light has a longer path length than the upward emitted light, so it is much redder when it leaves the surface of the ocean (Figure 3). This was clear from inspection of the depth dependent radiance distributions from the HydroLight output. When comparing the two bottom types, there is a larger wavelength shift with a sea grass bottom, as it is more absorptive in the peak bioluminescence wavelength than a coral sand bottom.

\subsection{Neural Net Predictions}

Two neural net models were trained for each of the eight conditions shown in Figure 2; one for predicting the geometric depth of the bioluminescent source and one for predicting $\tau$ of the bioluminescent source. Each model was trained on a randomly selected $50 \%$ of the spectra and their associated depths and then used to predict the remaining $50 \%$ of the data. The neural nets were able to predict the depth of the bioluminescent layer within 0.5 meters for each of the eight conditions (diagonal in Table 1a). In addition, there were a few cases where a neural network trained on one type of condition performed well predicting a different condition. For example, a neural net model trained on a water column with $50 \%$ of the natural IOPs with a sea grass bottom was able to predict the bioluminescent source depth from a water column with $50 \%$ of the natural IOPs with a coral sand bottom. In our analysis, neural net models performed well when applied to water columns with similar IOPs, indicating that even at low magnitude IOPs, bottom type did not appear to play as large a role as IOPs in accuracy of the prediction. Also, when a neural net model was trained on all conditions, it was able to predict well in all cases. Table $1 \mathrm{~b}$ shows that $\tau$ was best predicted when the neural net was asked to predict the $\tau$ of a bioluminescent source from the same condition on which the neural net was trained. Unlike depth, $\tau$ predictions are not directly comparable between conditions because $\tau$ depends also on the IOPs above the source. However, as was the case with the results in Table 1a, neural nets gave good predictions of $\tau$ from other conditions with similar IOPs. 
Neural net models were also trained on the year 2000 data and used to predict the year 2001 data. For each of the eight conditions, the neural net models were able to predict the depth (Figure 4) and $\tau$ (Figure 5) of the bioluminescent layer on the basis of the previous year's data. However, the neural net models underestimated $\tau$ for the larger $\tau$ values.

\subsection{Wavelength Reduction}

\section{--- INSERT FIGURE 6 ---}

Using the prediction of year 2001 data from year 2000 data as a starting point, we systematically reduced the number of wavelengths of water-leaving radiance that we allowed to neural net model to train with, in order to determine how many wavelengths are needed to make an accurate prediction of depth or $\tau$. We tested with 37, 19, 10, 5, 3, and 2 wavelengths and evaluated the models' performance using an RMS error statistic. All models predicting depth performed well, with as little as three wavelengths of water-leaving radiance (RMS $<1 \mathrm{~m}$, Figure 6a). Only under conditions where IOPs were larger than $10 \%$ of natural conditions were two wavelengths of water-leaving radiance insufficient for accurate prediction. RMS errors of models predicting $\tau$ are not directly comparable because $\tau$ is dependent on the IOPs, which is changing across the tested conditions. Therefore we also examined the percentage error of $\tau$. Similar to the result achieved with the models predicting depth, the models performed well in predicting $\tau$ until the wavelength number was reduced to less than three. With only two wavelengths in the model, most neural networks continued to perform well; however, errors in the neural network trying to predict all conditions increased dramatically.

\section{Discussion}

The spectral shape of a light source in the ocean carries with it the integrated history of its absorption and scattering. However, it is often difficult to determine the source location of the light, given the number of different absorption and scattering regimes through which the light could have passed. Solutions to inverse problems such as these are often poorly constrained [McCormick, 1992]. Therefore assumptions and approximations must be made, or information about the environment of the internal light source is needed to reduce the degrees of freedom and approach a solution [Tao et al., 1994]. When the problem is approached analytically, a detailed understanding of the mechanisms is important in determining which assumptions are valid. This is why many optical algorithms must be "locally tuned" before they can be reliably applied [Platt et al., 1995]. Tuning is needed not necessarily because of an incomplete understanding of the forces affecting radiative transfer, but because analytic approaches to inverse problems often demand supplementary information about the region which is not always available for the solution. However, if the interest in the inverse problem is correlative rather than mechanistic, then we can employ a statistical approach in trying to extract the important information from the spectrum. For example, our interest is not in understanding the mechanistic relationship between the internal light source and its spectrum, but rather using the spectrum as a proxy for a particular characteristic of the light source (namely its vertical position in the water column). This is not to say that the mechanistic relationship between a bioluminescent light source and its water-leaving radiance is not useful, conversely; for the statistical approach to be meaningful, it must assume 
an underlying mechanistic relationship. In addition, it is the mechanistic relationship that gives clues to which statistical approach should be used.

One of the common critiques of using a statistical approach like a neural net is that it operates like a "black box." In other words, even though neural nets can be used to predict complex patterns like those of inverse radiative transfer problems, nothing new is learned about the underlying mechanisms of the problem, therefore they offer no real progress. However, this critique is a red herring, as neural nets propose to understand in a statistical way, not a mechanistic way. Furthermore, analysis of neural net performance with different inputs does provide insight into how much information is needed to solve the problem. The number of wavelengths needed to solve complex inverse problems is important to the remote sensing community because it affects both sensor and study design. In our study, the prediction ability of the neural nets was basically the same when three wavelengths were used (Figure 6); however, the error decreased when more wavelengths were included in the model. Figures 5 and 6 also highlight the power and transportability of this approach. Due to the natural variation in attenuation at the LEO study site and because we artificially varied the IOPs, the $\tau$ values ranged from $0.1-55$ (Figure 5a). Despite this, the neural nets still performed well, even with only a few wavelengths of $\mathrm{BL}_{\mathrm{w}}$ used as input (Figures $6 \mathrm{~b}$ and $6 \mathrm{c}$ ). This indicates that this approach is transportable to almost any natural water environment. For example, using an approximate water column spectral attenuation of $0.06 \mathrm{~m}^{-1}$ at $490 \mathrm{~nm}$ from the Biowatt experiment in the Sargasso Sea [Smith et al., 1989], and applying the RMS errors in $\tau$ we modeled at the $10 \%$ IOP level (simulating clearer, less variable ocean waters), we estimate uncertainty in the location of the bioluminescent source depth to be $\sim 5 \mathrm{~m}$. It is fortuitous for naval applications that the size of potential bioluminescent stimulations scale with optical loads in oceanic systems, with presumably larger scale stimulations occurring in clear open ocean environments and smaller scale bioluminescent events occurring in more turbid coastal waters. Results from this study show the RMS errors of the prediction scale with the optical load (i.e., $\tau$ ), illustrating that this modeling approach is directly relevant to the applied interests of bioluminescence in the ocean.

The shift from an analytic to a neural net approach to solving inverse radiative transfer problems such as the one presented here also carries with it a different set of assumptions. Whereas analytic approaches make certain assumptions about the underlying mechanics of radiative transfer, the assumption of the neural net is that the training data set captures and sufficiently approximates all of the possible radiative transfer solutions in the test data set. This is illustrated in Tables 1a and 1b, where the diagonals have the lowest error rates compared to the rest of the error matrix. In Figures 4 and 5, the year-to-year prediction was successful because, by and large, the scope of solutions in 2000 was similar to that of 2001.

In an ocean that is poorly sampled, satisfying the assumptions of a neural net approach to inverse radiative transfer is difficult. Neural nets in many cases have been rightly rejected as viable tools in oceanography because their assumptions could not be met with any degree of confidence. However, the emergence of new technologies and synoptic data collection in the ocean allow the assumptions of a neural net approach to be satisfied and used successfully [Kocak et al., 1999; Schiller and Doerffer, 1999; Slade et al., 2003]. In the last ten years, the way we observe the ocean has been revolutionized [Schofield and Glenn, 2004]. Computing ability and autonomous technology provide data in volumes and quality never before achieved. 
Therefore the assumptions of neural net approaches to solving complex inverse radiative transfer problems can be met, making them a viable and valuable option for ocean researchers who seek to use water-leaving radiance data as proxies for studies not directly related to the mechanics of radiative transfer.

The success of neural networks in recovering the depth of bioluminescent source layers in the horizontally homogenous ocean simulated here warrants similar investigations for point sources of bioluminescence, which were studied by [Gordon, 1987] using Monte Carlo simulations. For point sources, the geometry is inherently three-dimensional, and the waterleaving radiance varies with horizontal distance from the embedded source. In such cases, the spatial pattern as well as the spectral dependence of the water-leaving radiance carries information about the depth of the source, which helps to constrain the inversion. In addition, it should be noted that because our results are modeled, our analysis does not take into account light contamination issues, atmospheric correction problems, or sensitivity of the result to the resolution of the IOPs. Whether or not these issues are major problems for the neural net is unknown. It is conceivable alternate forms of a neural net model would not be severely hindered by these issues, as long as it was trained on measured $\mathrm{BL}_{\mathrm{w}}$ that included atmospheric and light contamination errors. These issues should be addressed in future projects.

\section{Summary}

The paired papers we present here combine direct observation of hydrography, bioluminescence, IOPs and the modeling of $\mathrm{BL}_{\mathrm{w}}$. We show that the bioluminescent community responds quickly to a changing environment, and is often concentrated along density gradients. In addition, the IOPs of the water column significantly change the spectral quality of the bioluminescent signal. However, despite the variability in bioluminescent potential, hydrography and IOPs, the depth and $\tau$ of the bioluminescent source was still able to be elucidated with by simple neural networks that were based only on $\mathrm{BL}_{\mathrm{w}}$. Equally important, the range of $\mathrm{BL}_{\mathrm{w}}$ is shown here to be detectable, and may be used as a tool for researchers focused on the vertical structure and ecology of bioluminescent organisms.

\section{Acknowledgments}

The authors wish to thank the Ocean Partnership Program (N00014-97-1-1019), the Office of Naval Research COMOP and HyCODE programs (N00014-97-0767 and N00014-990196), and the NSF EEGLE program (OCE-9727341 and OCE-9727342). We also want to thank Wayne Slade for valuable discussions about neural nets. Great thanks also go to the supporters (under ONR contract N00014-04-C0213 to author C.D.M.) of and participants in the 2004 Summer Courses in Ocean Optics and Biogeochemistry: "Radiative Transfer and Remote Sensing," at the University of Maine Darling Marine Center, where many of the ideas presented here were hatched during great discussions with plenty of friends and food. Finally, we would like to thank the great state of New Jersey for its continuing support. 


\section{References}

Abrahams, M. V., and L. D. Townsend (1993), Bioluminescence in dinoflagellates: A test of the burglar alarm hypothesis, Ecology, 74, 258-260.[CrossRef]

Burkenroad, M. D. (1943), A possible function of bioluminescence, J. Mar. Res., 5, 161-164.

Gordon, H. R. (1987), Bio-optical model describing the distribution of irradiance at the sea surface resulting from a point source embedded in the ocean, Appl. Opt., 26, 4133-4148.

Herren, C. M., S. H. D. Haddock, C. Johnson, M. A. Moline, C. M. Orrico, and J. F. Case (2005), A multi-platform bathyphotometer for fine-scale, coastal bioluminescence research, Limnol. Oceanogr. Methods, 3, 247-262.

Herring, P. J. (1987), Systematic distribution of bioluminescence in living organisms, J. Bioluminesc. Chemiluminesc., 1, 147-163.[CrossRef]

Kirk, J. T. O. (1994), Light and Photosynthesis in Aquatic Ecosystems, 509 pp., Cambridge Univ. Press, Cambridge, U.K.

Kocak, D. M., N. D. V. Lobo, and E. A. Widder (1999), Computer vision techniques for quantifying, tracking, and identifying bioluminescent plankton, IEEE J. Oceanic Eng., 24, 8195.[CrossRef]

Lapota, D. (2003), Buoy-mounted bioluminescence sensor (BioBuoy) for special operations, Tech. Doc. 3155, pp. 142-145, Space and Naval Warfare Cent., San Diego, Calif.

Lapota, D. (2005), Night time surveillance of harbors and coastal areas using bioluminescence camera and buoy systems, in Photonics for Port and Harbor Security, edited by M. J. DeWeert and T. T. Saito, Proc. SPIE Int. Soc. Opt. Eng., 5780, 128-137.

Leathers, R. A., C. S. Roesler, and N. J. McCormick (1999), Ocean inherent optical property determination from in-water light field measurements, Appl. Opt., 38, 5096-5103.

Lynch, R. V. (1981), Patterns of bioluminescence in the oceans, NRL Rep. 8475, 32 pp., Naval Res. Lab., Washington, D. C.

McCormick, N. J. (1992), Inverse radiative transfer problems: A review, Nucl. Sci. Eng., 112, 185-198.

Mobley, C. D., and L. K. Sundman (2001a), HydroLight 4.2 Technical Documentation, Sequoia Sci., Inc., Mercer Island, Wash. (Available at http://www.HydroLight.info)

Mobley, C. D., and L. K. Sundman (2001b), HydroLight 4.2 Users' Guide, Sequoia Sci., Inc., Mercer Island, Wash. (Available at http://www.HydroLight.info) 
Moline, M. A., et al. (2005), Remote Environmental Monitoring UnitS: An autonomous vehicle for characterizing coastal environments, J. Atmos. Oceanic Technol., 22, 1798-1809.[CrossRef]

Moline, M. A., M. J. Oliver, C. D. Mobley, L. Sundman, T. Bensky, T. Bergmann, W. P. Bissett, J. Case, E. H. Raymond, and O. M. E. Schofield (2007), Bioluminescence in a complex coastal environment: 1. Temporal dynamics of nighttime water-leaving radiance, J. Geophys. Res., doi:10.1029/2007JC004138, in press.

Morin, J. G. (1983), Coastal bioluminescence patterns and functions, Bull. Mar. Sci., 33, 787817.

Nealson, K. H. (1993), Bacterial bioluminescence: Three decades of enlightenment, Naval Res. Rev., 45, 13-30.

Petzold, T. J. (1972), Volume scattering functions for selected ocean waters, Tech. Rep. SIO, pp. 72-78, Scripps Inst. of Oceanogr., San Diego, Calif.

Platt, T., S. Sathyendranath, A. Longhurst, T. R. S. Wilson, J. Woods, and T. Takahashi (1995), Remote sensing of primary production in the ocean: Promise and fulfilment [and discussion], Philos. Trans. Biol. Sci., 348, 191-202.[CrossRef]

Pope, R., and E. Fry (1997), Absorption spectrum (380-700 nm) of pure water, II, Integrating cavity measurements, Appl. Opt., 36, 8710-8723.

R Development Core Team (2006), R: A language and environment for statistical computing, Found. for Stat. Comput., Vienna.

Schiller, H., and D. Doerffer (1999), Neural network for emulation of an inverse model operational derivation of Case II water properties from MERIS data, Int. J. Remote Sens., 20, 1735-1746.[CrossRef]

Schofield, O., and S. Glenn (2004), Introduction to special section: Coastal Ocean Observatories, J. Geophys. Res., 109, C12S01, doi:10.1029/2004JC002577.[AGU]

Slade, W. H., Jr., R. L. Miller, H. Ressom, and P. Natarajan (2003), Ensemble neural network methods for satellite-derived estimation of chlorophyll, in Proceedings of the International Joint Conference on Neural Networks, pp. 547-552, Inst. of Electr. and Electron. Eng., New York.[CrossRef]

Smith, R. C., J. Marra, M. J. Perry, K. S. Baker, E. Swift, E. Buskey, and D. A. Kiefer (1989), Estimation of a photon budget for the upper ocean in the Sargasso Sea, Limnol. Oceanogr., 34, $1673-1693$.

Sundman, L. K., R. Sanchez, and N. J. McCormick (1998), Ocean optical source estimation with widely spaced irradiance measurements, Appl. Opt., 37, 3793-3803. 
Tao, Z., N. J. McCormick, and R. Sanchez (1994), Ocean source and optical property estimation from explicit and implicit algorithms, Appl. Opt., 33, 3265-3275.

Venables, W. N., and B. D. Ripley (2002), Modern Applied Statistics With S, 4th ed., 495 pp., Springer, New York. 


\section{Figures}

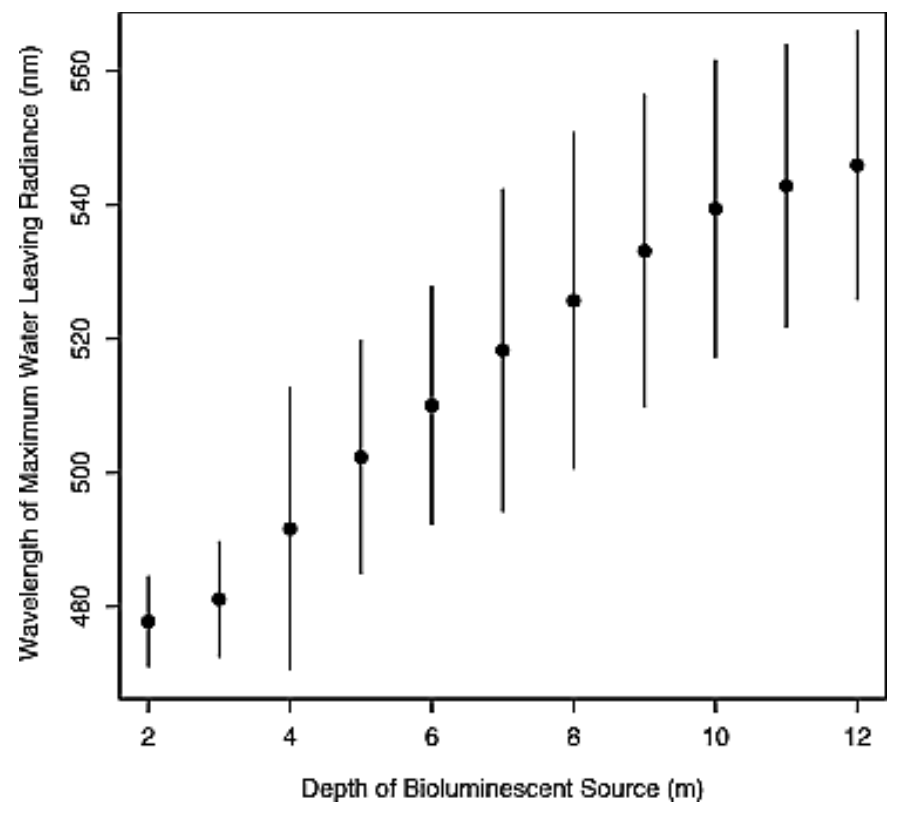

Figure 1. The wavelength of maximum $\mathrm{BL}_{\mathrm{w}}$ has a positive relationship to the depth to the bioluminescent source under natural conditions at the LEO study site, indicating that the spectral shape of $\mathrm{BL}_{\mathrm{w}}$ contains depth information. The points indicate the mean wavelength for each depth, and the bars represent two standard deviations. This figure summarizes the results from Figures $6 \mathrm{~b}$ and $7 \mathrm{~b}$ in part 1 of this paper [Moline et al., 2007]. The errors associated with a simple linear model relating the wavelength of maximum $\mathrm{BL}_{\mathrm{w}}$ indicate a more complex analysis is needed to predict the depth of the bioluminescent source from $\mathrm{BL}_{\mathrm{w}}$. Enhanced EPS [125 KB] 


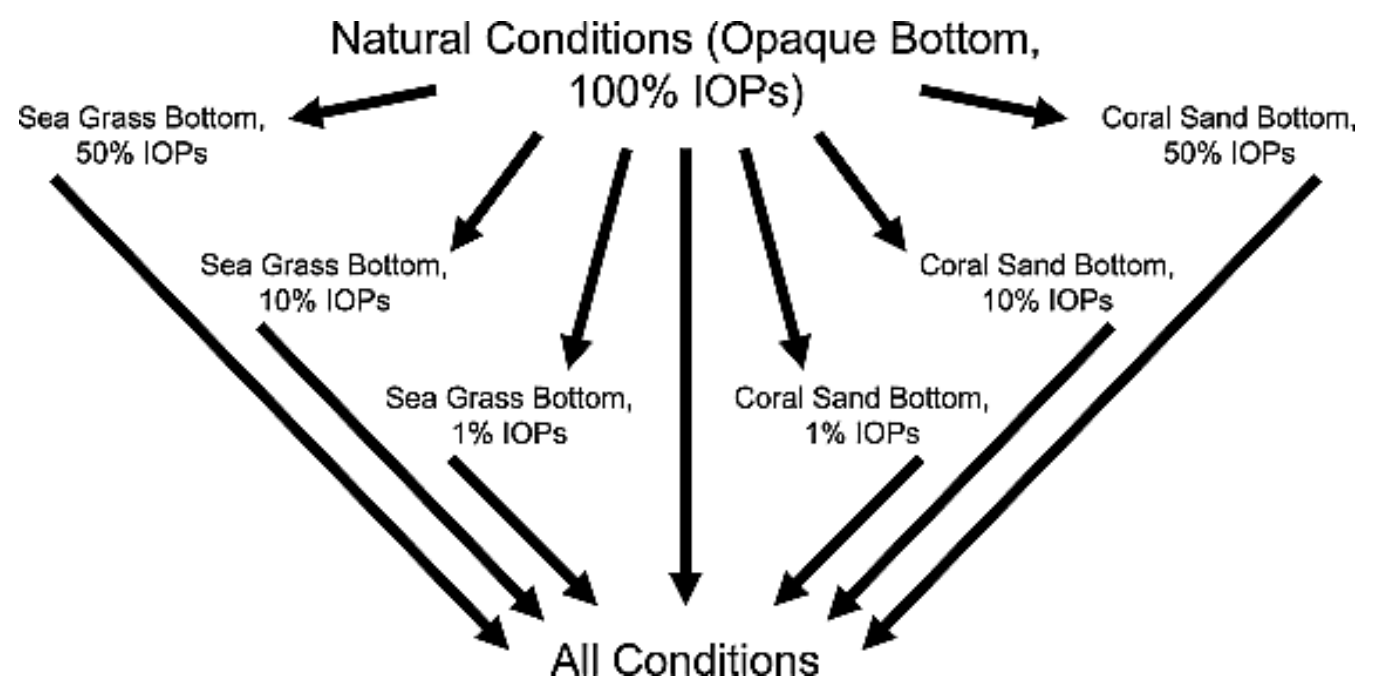

Figure 2. Flow diagram showing the modulation of the natural conditions measured at LEO-15. IOPs were reduced to $50 \%, 10 \%$, and $1 \%$ of their original. In addition, a sea grass and coral sand bottom were added to determine what effect these had on spectral $\mathrm{BL}_{\mathrm{w}}$. These individual conditions were also pooled into general category called "All Conditions." Enhanced EPS [242 $\mathrm{KB}]$ 


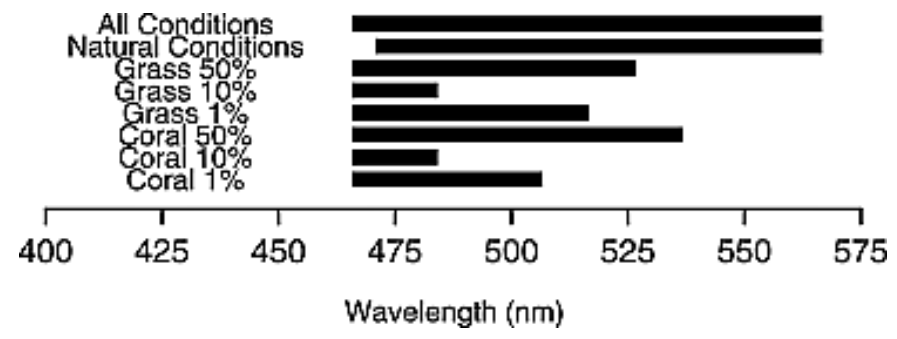

Figure 3. The range of wavelengths of maximum $\mathrm{BL}_{\mathrm{w}}$ for the various optical and bottom type conditions. The natural conditions show that the wavelength of maximum emission has a range of nearly $100 \mathrm{~nm}$. As IOPs are decreased, the range of maximum wavelength of emission also decreases, with the exception of the $1 \%$ IOP condition where bioluminescent signal that emitted downward is reflected off the bottom and then leaves the water surface. Enhanced EPS [99 KB] 


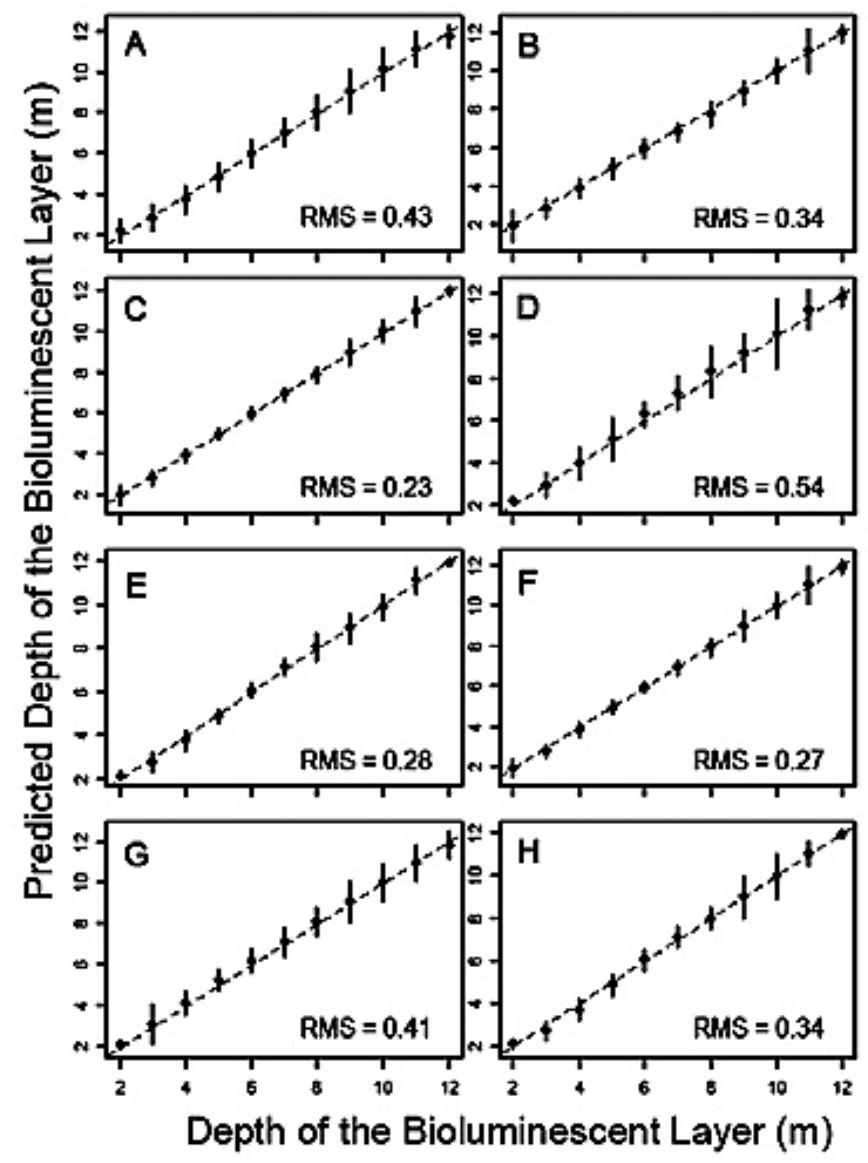

Figure 4. According to the eight conditions described in Figure 2, eight neural nets were trained on the bioluminescent water-leaving radiance and their associated depths collected in 2000, and were used to predict the depth of the bioluminescent layers in 2001 on the basis of their $\mathrm{BL}_{\mathrm{w}}$. For all eight conditions, the RMS error was less than $1 \mathrm{~m}$. Dashed line is the 1:1 line. Because the depth we trained the neural net on was discreet, we plotted the mean and the second standard deviation intervals for ease of interpretation. (a) All Conditions; (b) Natural Conditions; (c) Sea Grass Bottom-50\% IOPs; (d) Sea Grass Bottom-10\% IOPs; (e) Sea Grass Bottom-1\% IOPs; (f) Coral Sand Bottom-50\% IOPs; (g) Coral Sand Bottom-10\% IOPs; (h) Coral Sand-1\% IOPs. Enhanced TIF [388 KB] 


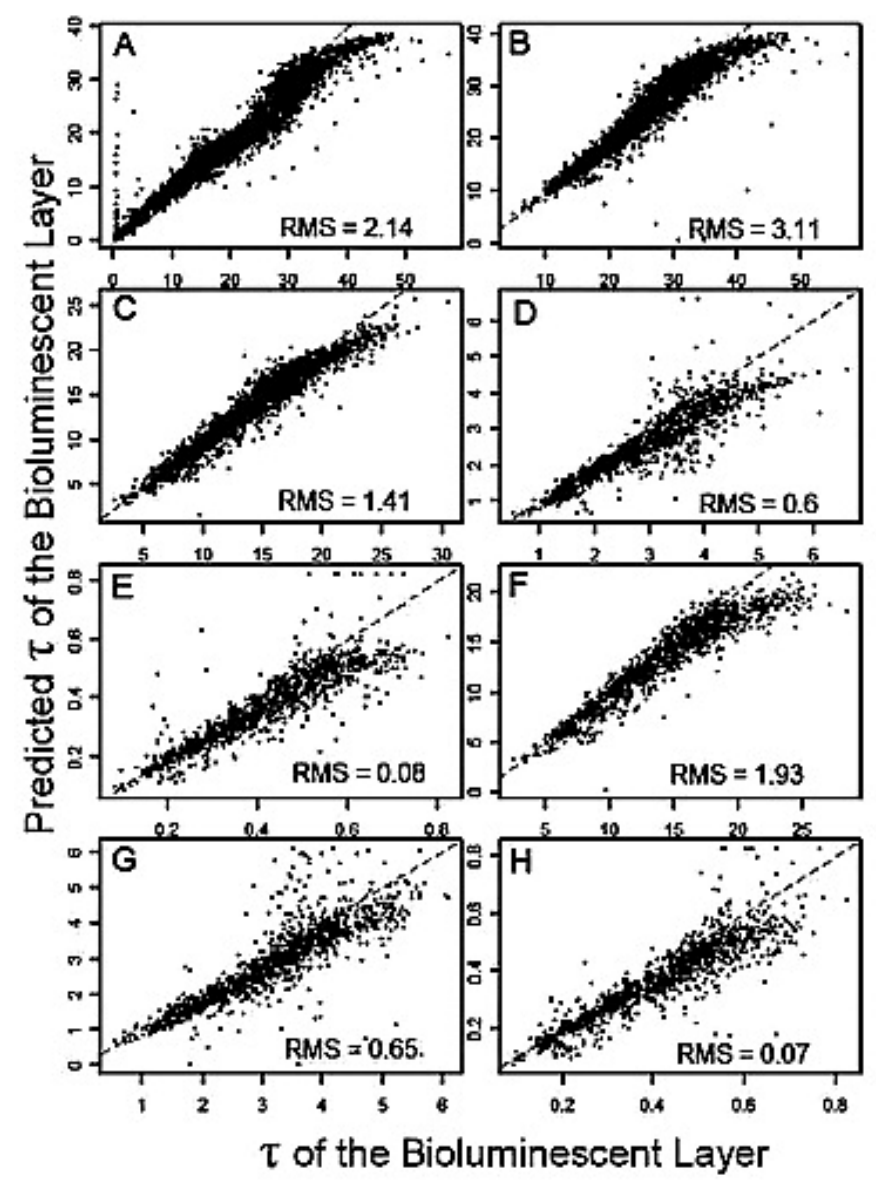

Figure 5. According to the eight conditions described in Figure 2, eight neural nets were trained on $\mathrm{BL}_{\mathrm{w}}$ and their associated $\tau$ from year 2000, and they were then used to predict the $\tau$ of the bioluminescent layers in year 2001. For all eight conditions the RMS error was small compared to the range over which the neural net was asked to predict. Dashed line is the 1:1 line. (a) All Conditions; (b) Natural Conditions; (c) Sea Grass Bottom-50\% IOPs; (d) Sea Grass Bottom-10\% IOPs; (e) Sea Grass Bottom-1\% IOPs; (f) Coral Sand Bottom-50\% IOPs; (g) Coral Sand Bottom10\% IOPs; (h) Coral Sand-1\% IOPs. Enhanced TIF [837 KB] 

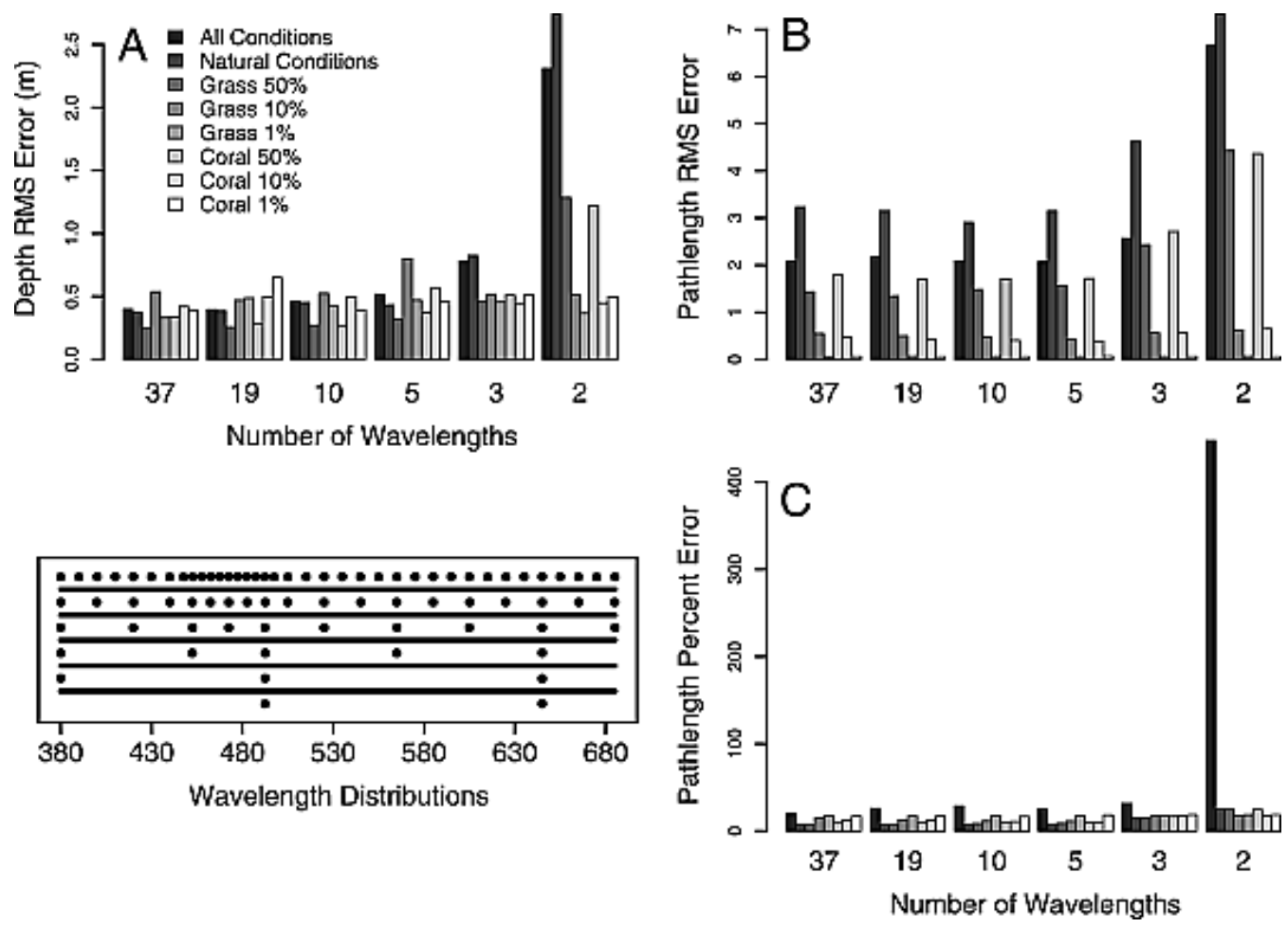

Figure 6. (a) RMS error of depth prediction from training on the year 2000 data and predicting the 2001 data for various numbers of spectral wavelengths used in the model. For all eight conditions, as little as three wavelengths from the spectral water-leaving radiance are needed for robust prediction of the depth of the bioluminescent layer. (b) RMS error of $\tau$ prediction from training on the year 2000 data and predicting the 2001 data for various numbers of spectral wavelengths used in the model. Errors are not directly comparable because $\tau$ also depends on the attenuation, which changed significantly between the eight conditions tested. (c) Path length errors as a percentage show that errors for all conditions are relatively low when more than five wavelengths are used in a neural net model. Errors increase slightly with three and two wavelengths in a neural net model in most conditions, but increase significantly with neural nets trained on all conditions. The bottom left panel shows wavelengths used in the reduction procedure. At each reduction step the number of wavelengths was reduced by $\sim 50 \%$ and alternate wavelengths were chosen. Enhanced EPS [311 KB] 


\section{Tables}

Table 1a. RMS Errors of Neural Net Prediction of Depth From the Eight Conditions Specified in Figure $2^{\mathrm{a}}$

\begin{tabular}{|c|c|c|c|c|c|c|c|c|c|}
\hline \multirow{2}{*}{} & \multicolumn{7}{|c|}{ Predictor } \\
\cline { 2 - 10 } & $\begin{array}{c}\text { All } \\
\text { Conditions }\end{array}$ & $\begin{array}{c}\text { Natural } \\
\text { Conditions }\end{array}$ & $\begin{array}{c}\text { Grass } \\
\mathbf{5 0 \%}\end{array}$ & $\begin{array}{c}\text { Grass } \\
\mathbf{1 0 \%}\end{array}$ & $\begin{array}{c}\text { Grass } \\
\mathbf{1 \%}\end{array}$ & $\begin{array}{c}\text { Coral } \\
\mathbf{5 0 \%}\end{array}$ & $\begin{array}{c}\text { Coral } \\
\mathbf{1 0 \%}\end{array}$ & $\begin{array}{c}\text { Coral } \\
\mathbf{1 \%}\end{array}$ \\
\hline Response & \multicolumn{7}{|c|}{} \\
\hline $\begin{array}{c}\text { All } \\
\text { conditions }\end{array}$ & 0.38 & 4.04 & 2.44 & 3.63 & 3.62 & 2.18 & 1.75 & 3.91 \\
\hline $\begin{array}{c}\text { Natural } \\
\text { conditions }\end{array}$ & 0.36 & 0.23 & 2.04 & 5.77 & 6.35 & 2.00 & 2.84 & 6.25 \\
\hline $\begin{array}{c}\text { Grass } \\
50 \%\end{array}$ & 0.30 & 2.17 & 0.21 & 3.71 & 3.37 & 0.29 & 1.59 & 4.19 \\
\hline $\begin{array}{c}\text { Grass } \\
10 \%\end{array}$ & 0.47 & 5.27 & 2.40 & 0.34 & 1.05 & 1.98 & 0.80 & 2.05 \\
\hline Grass 1\% & 0.35 & 6.14 & 3.76 & 1.05 & 0.23 & 3.04 & 1.26 & 1.35 \\
\hline Coral 50\% & 0.35 & 2.06 & 0.31 & 3.79 & 3.16 & 0.27 & 1.58 & 3.96 \\
\hline Coral 10\% & 0.39 & 5.12 & 2.02 & 0.78 & 0.74 & 1.50 & 0.30 & 1.34 \\
\hline Coral 1\% & 0.41 & 6.09 & 3.62 & 1.91 & 1.22 & 2.72 & 1.21 & 0.24 \\
\hline
\end{tabular}

${ }^{\mathrm{a}}$ The columns represent eight different models trained on the eight different conditions to predict depth $(\mathrm{m})$. The rows represent the condition being predicted by the model trained on conditions specified in the column. Values in the table are RMS errors of the depth prediction (m). Bold values indicate that the neural net model could, on average, predict the depth of a bioluminescent source light on the basis of its water-leaving radiance within $1 \mathrm{~m}$. 
Table 1b. Neural Net Prediction of $\tau$ From the Eight Conditions Specified in Figure $2^{\mathrm{a}}$

\begin{tabular}{|c|c|c|c|c|c|c|c|c|}
\hline & \multicolumn{8}{|c|}{ Predictor } \\
\hline & $\begin{array}{c}\text { All } \\
\text { Conditions }\end{array}$ & $\begin{array}{c}\text { Natural } \\
\text { Conditions }\end{array}$ & $\begin{array}{c}\text { Grass } \\
50 \%\end{array}$ & $\begin{array}{l}\text { Grass } \\
10 \%\end{array}$ & $\begin{array}{c}\text { Grass } \\
1 \%\end{array}$ & $\begin{array}{c}\text { Coral } \\
50 \%\end{array}$ & $\begin{array}{c}\text { Coral } \\
10 \%\end{array}$ & $\begin{array}{c}\text { Coral } \\
1 \%\end{array}$ \\
\hline \multicolumn{9}{|l|}{ Response } \\
\hline $\begin{array}{l}\text { All } \\
\text { conditions }\end{array}$ & 1.10 & 3.25 & 16.35 & 26.25 & 20.12 & 15.21 & 27.69 & 23.62 \\
\hline $\begin{array}{c}\text { Natural } \\
\text { conditions }\end{array}$ & 1.62 & 1.56 & 22.57 & 27.72 & 14.51 & 23.18 & 22.30 & 14.63 \\
\hline Grass $50 \%$ & 6.47 & 6.58 & 0.93 & 12.94 & 5.28 & 1.23 & 12.83 & 7.22 \\
\hline Grass $10 \%$ & 2.49 & 2.24 & 2.17 & 0.31 & 2.47 & 2.10 & 0.38 & 1.24 \\
\hline Grass $1 \%$ & 0.38 & 0.32 & 0.35 & 0.18 & 0.05 & 0.35 & 0.19 & 0.07 \\
\hline Coral $50 \%$ & 6.45 & 6.58 & 1.16 & 11.91 & 5.30 & 1.21 & 11.75 & 6.96 \\
\hline Coral 10\% & 2.49 & 2.27 & 2.16 & 0.37 & 0.88 & 1.97 & 0.28 & 1.22 \\
\hline Coral $1 \%$ & 0.38 & 0.34 & 0.36 & 0.22 & 0.14 & 0.33 & 0.22 & 0.05 \\
\hline
\end{tabular}

